\title{
Blind Spots in Fighting the Outbreak of Coronavirus Disease 2019
}

\author{
Lanjing Zhang* \\ Department of Pathology, Princeton Medical Center, Plainsboro, NJ, USA; Department of Biological Sciences, Rutgers University, \\ Newark, NJ, USA; Rutgers Cancer Institute of New Jersey, New Brunswick, NJ, USA; Department of Chemical Biology, \\ Ernest Mario School of Pharmacy, Rutgers University, Piscataway, NJ, USA
}

\begin{abstract}
The recent outbreak of 2019 novel coronavirus infection has involved more than 110,000 people and 105 countries. Many efforts have been made to prevent, contain and treat the related disease (named as coronavirus disease 2019). However, many blind spots might not yet receive needed attention. I here discuss eight blind spots that may interest related parties. If these issues remain outstanding, they will likely lead to many severe harms to the public, healthcare providers and the economy. Additional research is therefore needed to better understand and address these blind spots in fighting the outbreak of coronavirus disease 2019.
\end{abstract}

Recently, the outbreak of 2019 novel coronavirus infection involved more than 113,702 people and 109 countries in the word, and has led to a public health emergency of international concern. ${ }^{1,2}$ Many efforts were rightly and timely made to fight the outbreak of 2019 novel coronavirus in China, which was recently named as Severe acute respiratory syndrome coronavirus 2 (SARS-CoV-2) by the Coronavirus Study Group, ${ }^{3}$ and Coronavirus disease 2019 (COVID-19) by the World Health Organization (WHO). ${ }^{4}$ A group of virologists in China also proposed to name the virus as human coronavirus 2019 (HCoV-19). ${ }^{5}$ Despite the differences in name, we observed orchestral actions from governments, health care providers, non-profit organizations and individual citizens in China and the other part of the world. As a result, the daily incidence of infection has been decreasing since February 1, 2020 according to the official websites ${ }^{6,7}$ and a trend analysis on the epidemiology of COVID-19.8,9 On the other hand, the rising incidence in Japan, Korea and Iran is alarming. ${ }^{2}$ A modelling study also shows that Africa might be vulnerable to the pandemic of COVID-19. ${ }^{10}$ Indeed, there were 10,566 COVID-19 cases in WHO member states outside of China on March 3, 2020, which was developed within the past 2 weeks. ${ }^{2}$ On March 3 alone, 8 new member states of the WHO (namely Andorra, Jordan, Latvia, Morocco, Portugal, Saudi Arabia, Senegal, and Tunisia) reported their first confirmed new case. $^{2}$ The daily incidence of COVID-19 outside of China has been higher than that in China since February 26, 2020 (459 versus 412). Thus, we should continue our undivided attention on the surveil-

Keywords: COVID-19; Viral infection; Epidemic; Coronavirus; Epidemiology. Abbreviations: COVID-19, coronavirus disease 2019; SARS-CoV-2, severe acute respiratory syndrome coronavirus 2; WHO, World Health Organization.

Received: February 26, 2020; Revised: March 04, 2020; Accepted: March 05, 2020

${ }^{*}$ Correspondence to: Lanjing Zhang, Department of Pathology, Princeton Medical Center, Plainsboro, NJ, USA. Tel: +1-609-853-6833, Fax: +1-609-853-6841,

E-mail: lanjing.zhang@rutgers.edu

How to cite this article: Zhang L. Blind Spots in Fighting the Outbreak of Coronavirus Disease 2019. Exploratory Research and Hypothesis in Medicine 2020;5(1):6-7. doi: 10.14218/ERHM.2020.00012. lance, prevention and control of COVID-19 on the global scale.

Several key questions have been asked regarding the pathogenicity and transmissibility characteristics of COVID-19. ${ }^{11}$ Many works have been focused on the trends and characteristics of the disease/viral infection. ${ }^{8,12,13}$ However, several blind spots in my view are noteworthy during the fight against the outbreak of COVID-19. If not prevented or insufficiently prepared for, those blind spots might cause unexpected long-lasting harms, and consume significant amount of resources.

Eight issues might be of our particular interest and concern:

1. Distrust and isolated actions among (potentially) involved countries. Due to reasonable worries about the international spread of the COVID-19, there is significant disruption of international trades, academic/educational exchanges and political collaborations. ${ }^{14,15}$ Such disruption may escalate in the near future if the spread of COVID-19 continues involving more countries. However, it is probably also the time when international collaborations are urgently and most needed. The hard-learned lessons and practical experiences in China in my view are of ultimate values in fighting this novel virus around the world. Probably more importantly, proper coordination among all (involved) countries should be encouraged and could result in better utilization of available resources at the global scale.

2. Performance of the diagnostic tests. We would need tests like HIV tests, which include a screening test of high sensitivity and a confirmatory test of high specificity. ${ }^{16,17}$

3. Changes and updates of the diagnostic criteria might be necessary as we better understand the dynamic and characteristics of COVID-19. But these changes and update should be kept as few as possible for the continuity of disease surveillance, and consistency in treatment protocols and outcome. One recent change of including clinically diagnosed cases in confirmed cases led to a significant increase in daily incidence of COVID-19 in Wuhan City and Hubei Province. ${ }^{6,7,9}$ It caused much confusion and anxiety among the health care providers and the public.

4. Cautions in rushing into approving drugs for 2019-nCoV. We should follow the preset stand protocols in assessing and approv- 
ing new drugs. The due diligence must not be jeopardized despite the urgent needs for these drugs because an approval of such drug will have long-lasting and profound effects in the world.

5. The care for non-infectious diseases may be deprived of needed resources. Largely due to lack of manpower and resources, one should anticipate and prepare for a significant rise in the deaths due to cardiovascular, pulmonary and other chronic diseases.

6. Psychologic issues of the general public, patients, patient-families, local officials and healthcare providers. A recent comment rightfully raise such a concern. ${ }^{18}$ If not timely and properly addressed, such a psychological issue will exhaust healthcare manpower, decrease morale and cause long-term psychological diseases among impacted individuals including patients, families and health care providers.

7. Documentation of related policies, implemented approaches and observed outcomes. This practice will be critical for tracking records, learning the lessons, enforce accountability and improving efficiency.

8. Information overload and errors in reporting. It is inevitable that the conventional media and social media will pour unprecedent amount of information to the public, who are eager to better understand the disease and current situation. The important and accountable information, however, might be buried in the information overload. Related parties should proactively reach out to the public to "advertise" credible information through new media. Errors in reporting or rumors are very difficult to identify and handle since they could be proven wrong only upon rigorous, long and time-consuming validation studies.

Another question of significant consequences is how to properly deal with the aftermath of the COVID-19 outbreak. Hopefully, the end of the COVID-19 outbreak will soon come. Many issues will emerge. Thus, experts in public health, socioeconomical, geopolitical and biomedical studies should work together to proactively articulate the related issues, discuss possible solutions and formulate feasible, yet impactful, action plans.

In summary, the eight blind spots may interest related parties. If these issues remain outstanding, they will likely lead to many severe harms to the public, healthcare providers and the economy. Additional research is therefore needed to better understand and address these blind spots in fighting the outbreak of coronavirus disease 2019.

\section{Acknowledgments}

The opinions expressed here are solely those of the author, and not of his affiliations.

\section{Funding}

None.

\section{Conflict of interest}

The author has no conflict of interest related to this publication.

\section{References}

[1] Zarocostas J. What next for the coronavirus response? Lancet 2020;395(10222):401. doi:10.1016/S0140-6736(20)30292-0.

[2] WHO. Coronavirus disease (COVID-2019) situation reports. Available from: https://www.who.int/emergencies/diseases/novel-coronavirus-2019/situation-reports/. Accessed Feb. 26, 2020.

[3] Gorbalenya AE, Baker SC, Baric RS, de Groot RJ, Drosten C, Gulyaeva $A A$, et al. Severe acute respiratory syndrome-related coronavirus: The species and its viruses - a statement of the Coronavirus Study Group. bioRxiv 2020. doi:10.1101/2020.02.07.937862.

[4] WHO. Coronavirus disease (COVID-19) outbreak. Available from: https://www.who.int/emergencies/diseases/novel-coronavirus-2019. Accessed February 23, 2020.

[5] Jiang S, Shi Z, Shu Y, Song J, Gao GF, Tan W, et al. A distinct name is needed for the new coronavirus. Lancet 2020. doi:10.1016/s01406736(20)30419-0.

[6] Wuhan Municipal Health Commission. Briefings on the viral disease [Chinese]. Available from: http://wjw.wuhan.gov.cn/front/web/list3rd/no/802. Accessed Feb. 23, 2020.

[7] National Health Commission of China. Briefings on the viral outbreak [Chinese]. Available from: http://www.nhc.gov.cn/xcs/yqtb/list_gzbd. shtml. Accessed Feb. 24, 2020.

[8] Xu J, Cheng Y, Yuan X, Li WV, Zhang L. Trends in epidemiology of 2019 novel coronavirus. Available from: https://github.com/thezhanglab/ coronavirus. Accessed Feb. 23, 2020.

[9] Xu J, Cheng Y, Yuan X, Li WV, Zhang L. Trends and prediction in daily incidence of novel coronavirus infection in China, Hubei Province and Wuhan City: an application of Farr law. medRxiv 2020:20025148. doi:10.1101/2020.02.19.20025148.

[10] Gilbert M, Pullano G, Pinotti F, Valdano E, Poletto C, Boëlle PY, et al. Preparedness and vulnerability of African countries against importations of COVID-19: a modelling study. Lancet 2020. doi:10.1016/ S0140-6736(20)30411-6.

[11] Munster VJ, Koopmans M, van Doremalen N, van Riel D, de Wit E. A Novel Coronavirus Emerging in China - Key Questions for Impact Assessment. N Engl J Med 2020;382(8):692-694. doi:10.1056/NEJMp2000929.

[12] Zhang R, Liu H, Li F, Zhang B, Liu Q, Li X, et al. Transmission and epidemiological characteristics of Novel Coronavirus (2019-nCoV) Pneumonia (NCP): preliminary evidence obtained in comparison with 2003-SARS medRxiv 2020:20019836(v3). doi:10.1101/2020.01.30.20019836.

[13] Li Q, Guan X, Wu P, Wang X, Zhou L, Tong Y, et al. Early Transmission Dynamics in Wuhan, China, of Novel Coronavirus-Infected Pneumonia. N Engl J Med 2020. doi:10.1056/NEJMoa2001316.

[14] DHS. 2020 Travel/Visa Restrictions. Available from: https://www.dhs. gov/news/2020/01/31/2020-travelvisa-restrictions. Accessed March 3, 2020.

[15] Government UK. Policy paper: Coronavirus action plan: a guide to what you can expect across the UK. Available from: https://www.gov. uk/government/publications/coronavirus-action-plan/coronavirusaction-plan-a-guide-to-what-you-can-expect-across-the-uk. Accessed March 3, 2020.

[16] Branson BM. HIV Diagnostics: Current Recommendations and Opportunities for Improvement. Infect Dis Clin North Am 2019;33(3):611628. doi:10.1016/j.idc.2019.04.001.

[17] Chen DJ, Yao JD. Comparison of turnaround time and total cost of HIV testing before and after implementation of the 2014 CDC/APHL Laboratory Testing Algorithm for diagnosis of HIV infection. J Clin Virol 2017;91:69-72. doi:10.1016/j.jcv.2017.04.004.

[18] Xiang YT, Yang Y, Li W, Zhang L, Zhang Q, Cheung T, et al. Timely mental health care for the 2019 novel coronavirus outbreak is urgently needed. Lancet Psychiatry 2020;7(3):228-229. doi:10.1016/S22150366(20)30046-8. 